\title{
NWI open Quality of newborn care: a health facility assessment in rural Ghana using survey, vignette and surveillance data
}

\author{
Linda Vesel, ${ }^{1}$ Alexander Manu, ${ }^{2}$ Terhi J Lohela, ${ }^{3}$ Sabine Gabrysch, ${ }^{4}$ \\ Eunice Okyere, ${ }^{2}$ Augustinus $\mathrm{H}$ A ten Asbroek, ${ }^{5}$ Zelee Hill, ${ }^{6}$ \\ Charlotte Tawiah Agyemang, ${ }^{2}$ Seth Owusu-Agyei, ${ }^{2}$ Betty R Kirkwood ${ }^{1}$
}

To cite: Vesel L, Manu A, Lohela TJ, et al. Quality of newborn care: a health facility assessment in rural Ghana using survey, vignette and surveillance data. BMJ Open 2013;3:e002326.

doi:10.1136/bmjopen-2012002326

- Prepublication history for this paper are available online. To view these files please visit the journal online (http://dx.doi.org/10.1136/ bmjopen-2012-002326).

Received 9 November 2012 Revised 9 March 2013 Accepted 12 March 2013

This final article is available for use under the terms of the Creative Commons Attribution Non-Commercial 2.0 Licence; see http://bmjopen.bmj.com

For numbered affiliations see end of article.

Correspondence to Dr Linda Vesel; linda.vesel@gmail.com

\section{ABSTRACT}

Objective: To assess the structural capacity for, and quality of, immediate and essential newborn care (ENC) in health facilities in rural Ghana, and to link this with demand for facility deliveries and admissions.

Design: Health facility assessment survey and population-based surveillance data.

Setting: Seven districts in Brong Ahafo Region, Ghana.

Participants: Heads of maternal/neonatal wards in all 64 facilities performing deliveries.

Main outcome measures: Indicators include: the availability of essential infrastructure, newborn equipment and drugs, and personnel; vignette scores and adequacy of reasons given for delayed discharge of newborn babies; and prevalence of key immediate ENC practices that facilities should promote. These are matched to the percentage of babies delivered in and admitted to each type of facility.

Results: $70 \%$ of babies were delivered in health facilities; $56 \%$ of these and $87 \%$ of neonatal admissions were in four referral level hospitals. These had adequate infrastructure, but all lacked staff trained in ENC and some essential equipment (including incubators and bag and masks) and/or drugs. Vignette scores for care of very low-birthweight babies were generally moderate-to-high, but only three hospitals achieved high overall scores for quality of ENC. We estimate that only $33 \%$ of babies were born in facilities capable of providing high quality, basic resuscitation as assessed by a vignette plus the presence of a bag and mask. Promotion of immediate ENC practices in facilities was also inadequate, with coverage of early initiation of breastfeeding and delayed bathing both below 50\% for babies born in facilities; this represents a lost opportunity.

Conclusions: Unless major gaps in ENC equipment, drugs, staff, practices and skills are addressed, strategies to increase facility utilisation will not achieve their potential to save newborn lives.

Trial registration: http://clinicaltrials.gov NCT00623337.

\section{ARTICLE SUMMARY}

\section{Article focus}

- To address a large evidence gap in the quality of newborn facility care.

- To assess the structural capacity for, and quality of, immediate and essential newborn care (ENC) in health facilities in rural Ghana, and to link this with demand for facility deliveries.

Key messages

- Key gaps in ENC equipment, drugs and/or personnel and essential life-saving actions were found in all facilities. We estimate that only $33.2 \%$ of babies born in facilities had access to high quality, basic resuscitation.

- Promotion of early initiation of breastfeeding and delayed bathing was inadequate for all facility births.

- A one-off ENC facility training course had very little impact on the quality of care provided.

Strengths and limitations of this study

- This paper addresses a major evidence gap regarding facility care of newborns in low-and-middle income countries and identifies the supply side components of facility newborn care that need to be strengthened in order to match the demand for services and to increase newborn survival.

- The Health Facility Assessment was largely based on self-reports. Vignettes were designed as purposely simplified evaluations of crucial, basic newborn care on the first day of life aiming to emphasise the most obvious gaps and test the best practice by asking about intended care, which may differ slightly from actual care and could overestimate quality.

\section{INTRODUCTION}

The 3.3 million newborn deaths that occur in the first month of life account for $41 \%$ of under-five mortality and are disproportionately concentrated in low-and-middle income countries (LMICs). ${ }^{1-3}$ The majority $(75 \%)$ 
occurs in the first week, particularly on the first day $(25-50 \%),{ }^{2}{ }^{4}$ and can be saved through simple, costeffective and low technology interventions. ${ }^{5}{ }^{6}$ WHO and the United Nations Children's Fund recommend home visits by trained community-based agents (CBA) to promote essential newborn care (ENC) practices and to assess and then treat or refer sick newborns as a strategy to save newborn lives in LMICs. ${ }^{7}$ However, this strategy does not address a large proportion of deaths that occur on the first day, such as those due to birth asphyxia and those that happen before the CBA has had a chance to visit. Furthermore, assessing and referring sick newborns can only save lives if they receive appropriate care when they reach health facilities.

Several studies have reported inadequacies in the quality of facility care for maternal and child health in LMICs. $^{8-11}$ However, few have focused on the quality of neonatal care. ${ }^{12} 13$ The latest Countdown report, taking stock of maternal, newborn and child survival, has highlighted a major gap in evidence regarding the quality of facility care for newborns in LMICs, both immediately after delivery and of sick newborns in the postnatal period. ${ }^{1} 14$

The objective of this paper was to address this evidence gap in rural Ghana and, in so doing, aim to impact positive change in newborn care and survival. This is carried out by (1) assessing the structural capacity and quality of immediate and ENC in health facilities in seven districts of the Brong Ahafo region and (2) linking these findings to data on demand for delivery and admission services by women and newborns residing in these districts using surveillance data from a previous trial (Newhints ${ }^{15}$ —clinicaltrials.gov, NCT00623337).

\section{METHODS}

\section{Setting}

This health facility assessment (HFA) was carried out in all health facilities serving mothers and babies in the seven districts in the Brong Ahafo region of central, rural Ghana: Kintampo North and South, Nkoranza North and South Tain, Techiman and Wenchi. They are situated in a forest-Savannah transitional zone. There are more than 120000 women of reproductive age with over 15000 live-births/year. ${ }^{15}$ The neonatal death rate in the area is $31 / 1000$ live-births. ${ }^{15}$ The Newhints trial, from which surveillance data are used, was conducted in these districts before HFA.

Newhints was an intervention designed to improve newborn survival through home visits by communitybased surveillance volunteers (CBSVs) to promote facility delivery and ENC practices, and to refer sick and very low-birth-weight (LBW) babies to health facilities. ${ }^{15}$ Mothers were encouraged to go straight to one of the four main district hospitals in Kintampo, Techiman, Nkoranza and Wenchi. These district hospitals were referral destinations for all other facilities within the study area. Health facility strengthening and assessment were not part of the Newhints trial.

There are a total of 86 facilities serving mothers and babies in the study area, 64 of which perform deliveries (figure 1). These include a regional hospital located outside the seven districts but acting as the regional referral centre, four main district hospitals, four other district hospitals-two in newly formed districts and two in adjoining districts which some women use-four private hospitals, 37 health centres, 12 private maternity homes and 24 clinics.
Figure 1 Hierarchy of health facilities in the Brong Ahafo Region, Ghana.

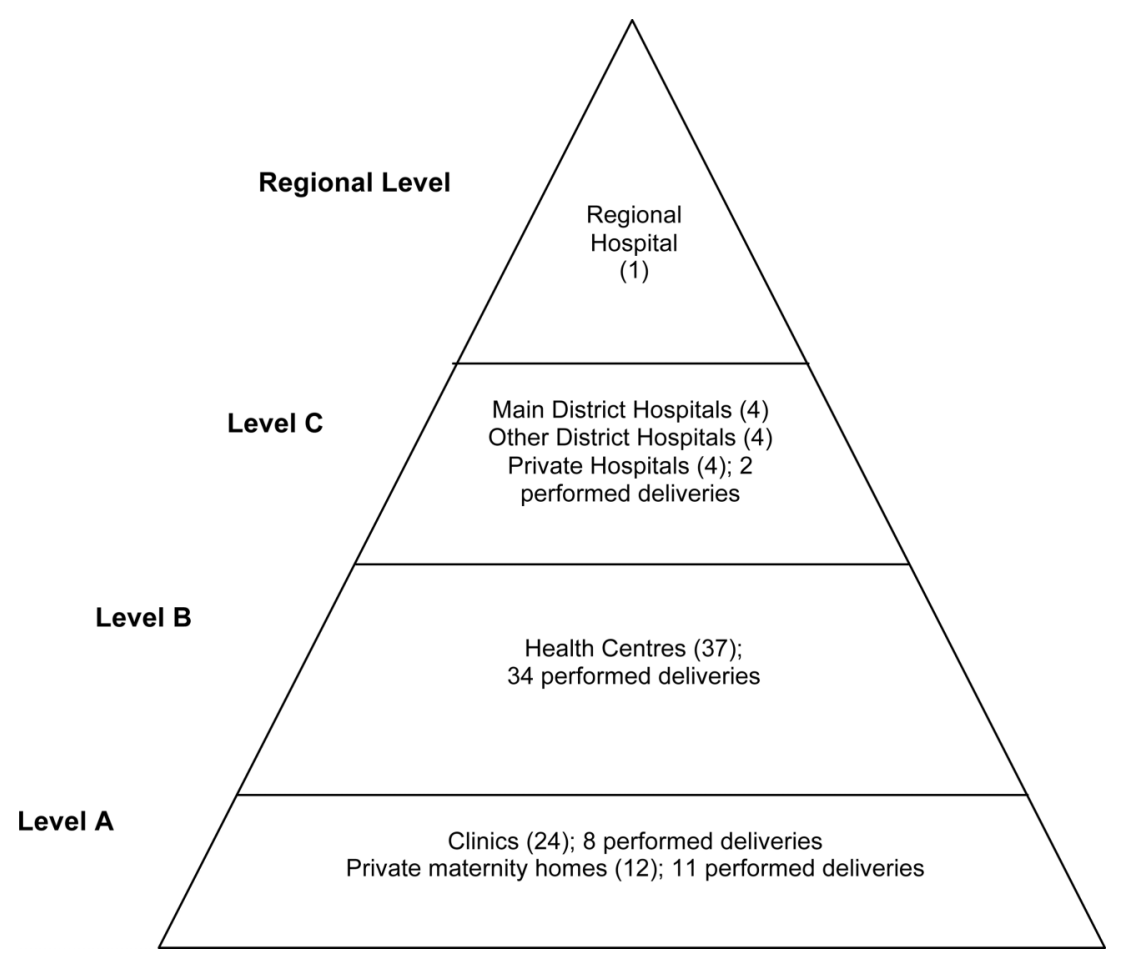


As a part of strengthening the facilities for the implementation of the Newhints intervention, the formative research carried out found inadequacies in the skills of the facility staff to care for the sick and vulnerable newborns referred to them. Thus, a training of facility staff in ENC was recommended. A WHO-sponsored national training of trainers' workshop on 'strengthening essential newborn care in health facilities' was organised in Accra. This was followed by two workshops at two of the main district hospitals in the study area for staff caring for sick newborns in the 14 topmost facilities where the majority of sick newborns were taken within the Newhints intervention area. ${ }^{15}$ Forty midwives and nurses from the largest facilities where most deliveries and sick newborns were taken for care received a 4-day ENC facility training using WHO's Pregnancy, Childbirth, Postpartum and Newborn Care (PCPNC) guidelines in two of the four main hospitals. ${ }^{16}{ }^{17}$ This training involved assessing newborns for danger signs, classifying their illness and treating or referring where needed. Practical sessions were conducted in the two hospitals as part of the training. ${ }^{15} 18$

\section{Health facility assessment: content and data collection}

HFA was conducted by a physician who was assisted by a research officer in all 86 facilities between June and December 2010. It was carried out with either the head of the facility's joint maternity/newborn ward or the most senior nurse/midwife available at the time of the interview.

HFA included sections on infrastructure (observed); antenatal, obstetric and newborn care provided; referral practices and vignettes to capture correct practices, one on ENC and two on obstetric care. Additional information captured from the first 11 facilities surveyed included: profile of human resources for managing sick newborns, reasons for delayed discharge of newborn babies and a vignette encapsulating care for very LBW babies. These 11 facilities were the four main district hospitals, and a purposive sample of other facilities focusing on the largest; these were one of the two new (other) district hospitals, the largest private hospital, two of the three largest maternity homes and three of the five largest health centres.

\section{Vignettes}

The two vignettes relating to newborn care are shown in tables 1 and 2. The ENC vignette comprised three parts (A, B and $\mathrm{C}$ ) on resuscitation, immediate newborn care of a stabilised baby, and thermal care. The very LBW vignette included two parts (A and B) on immediate care of very LBW babies and breastfeeding advice. The vignettes were read to each respondent, who was asked to describe the steps of care to be taken. ${ }^{19}$ The interviewer marked whether or not the respondent mentioned each of a list of best practice actions specified in

\section{Table 1 Vignette 1 (essential newborn care)}

\begin{tabular}{|c|c|}
\hline \multicolumn{2}{|l|}{ Vignette 1} \\
\hline Action & Score \\
\hline \multicolumn{2}{|c|}{$\begin{array}{l}\text { A woman in labour presents at this facility. The Fetal Heart Rate is more than } 160 \mathrm{bpm} \text {. On examination, her cervix } \\
\text { is fully dilated and the baby has the head in the perineum. The baby is delivered and is normal weight, but it does } \\
\text { not cry after delivery. What would you do for this baby? DON'T PROMPT! }\end{array}$} \\
\hline Dry quickly and vigorously & 2.66 \\
\hline Examine and suction the mouth & 2.16 \\
\hline Ensure extra warmth for the baby & 1.50 \\
\hline Use a bag and mask to ventilate if the baby does not cry after suctioning & 2.53 \\
\hline Apply cardiac massage if ventilation alone does not help & 1.16 \\
\hline Total score $(A)$ & 10 \\
\hline \multicolumn{2}{|l|}{ Suppose the resuscitation was successful, what would you do next? DON'T PROMPT! } \\
\hline Initiate breastfeeding immediately & 3.31 \\
\hline Keep in skin-to-skin contact with the mother & 4.34 \\
\hline Ensure and encourage hygiene & 2.34 \\
\hline Total Score $(B)$ & 10 \\
\hline \multicolumn{2}{|c|}{$\begin{array}{l}\text { During routine checking on the baby after about } 2 \mathrm{~h} \text {, you see the baby sleeping alone and the mother is sleeping } \\
\text { not in touch with baby. There is no covering on the baby since it wriggled out of the mother's cloth. What would } \\
\text { you do? DON'T PROMPT! }\end{array}$} \\
\hline Feel if the baby is too cold & 1.28 \\
\hline Take the temperature with a thermometer & 1.53 \\
\hline Give skin-to-skin care/kangaroo mother care by mother or put in incubator for rewarming & 3.94 \\
\hline Prevent draught in the room: check if windows are closed, switch off any fans in the ward & 1.41 \\
\hline Ask mother to breastfeed the baby & 1.84 \\
\hline Total Score $(\mathrm{C})$ & 10 \\
\hline Maximum score for the vignette & 30 \\
\hline
\end{tabular}


Table 2 Vignette 2 (care for very low-birth-weight babies)

\begin{tabular}{l} 
Vignette 2 \\
\hline Action \\
\hline A 17 -year-old woman pregnant for 8 months delivered a baby at home. A trained community volunteer weighed \\
the baby and found it to be $1.4 \mathrm{~kg}$. As a result, she referred the baby to your facility. What would you do for this baby? \\
DON'T PROMPT! \\
Detain for thorough examination \\
Ensure breastfeeding is established and provide support if necessary \\
Put the baby in an incubator OR skin-to-skin with the mother \\
Teach the mother to keep baby skin-to-skin/kangaroo mother care position (if in incubator, when taken out) \\
Check cord dressing and other potential sources of infection \\
Encourage and ensure hygiene in care \\
Total score (A) \\
Mother says the baby is not breastfeeding and was contemplating giving glucose solution. What would you do? \\
DON'T PROMPT! \\
Watch her breastfeed her baby and teach her good positioning and attachment \\
Examine the baby's mouth to ensure there are no anatomical deformities \\
If baby is not breastfeeding, teach her to express the milk and feed with a clean cup \\
Encourage infant formula only if exclusive breast milk is not possible and mother can afford \\
Educate her and encourage her to practice exclusive breastfeeding for the first 6 months of the baby's life \\
Total score (B) \\
Maximum score for the vignette \\
\hline
\end{tabular}

the WHO PCPNC guidelines. ${ }^{17}$ A score out of 10 was calculated for each part of the vignette based on the best practice actions mentioned. The points allocated to each action are shown in tables 1 and 2 and reflect expert opinion on the relative importance of the actions to immediate newborn survival. Sixteen experienced paediatricians were asked to allocate 10 points between the actions in each part to reflect their opinion on each action's importance. They were asked to allocate only whole or half points (eg, 2.5). Averages were then taken of the points they allocated to determine the score given to each action.

Scores of $8(80 \%)$ or more out of 10 could be achieved only if one of the lowest scoring items were missed; facilities achieving this level have therefore been categorised as high quality. Scores below 5 (50\%) occurred when at least two of the highest scoring items were missed; facilities that scored in this range were therefore categorised as low quality. Facilities in the middle $50-79 \%$ were categorised as moderate quality.

\section{Indicators of quality of care}

Quality of newborn care was assessed by classifying it into two components defined by Donabedian ${ }^{20}$ : (1) structure, characteristics of the setting in which care is administered and (2) process, the essential procedures in the delivery of care. Structural capacity indicators included the percentage of facilities with: (1) infrastructure indicators-a clean water source, reliable electricity, a fridge for storage of vaccines, drugs and blood and a sink with soap for hand washing; (2) ENC equipmentbag and mask, oxygen cylinder, suction machine/nasal aspirator, incubator, baby scale, cup to measure expressed breast milk and intravenous fluid and infusion set; (3) essential drugs necessary for care of the newborn -ampicillin, gentamicin, diazepam and dexamethasone and (4) profile of human resources for managing sick newborns. Process indicators included: (1) vignette scores, (2) whether or not each of the reasons were mentioned for the delayed discharge of newly delivered babies listed in the PCPNC guidelines and (3) two indicators capturing ENC practices that should be promoted by facilities: percentage of babies born in facilities where breastfeeding was initiated within $1 \mathrm{~h}$ of birth and percentage of babies born in facilities where bathing was delayed for at least $6 \mathrm{~h}$ based on surveillance data.

\section{Matching quality to demand}

Results from HFA were matched with the demand for heath facility services, using surveillance data from the Newhints trial on the number of deliveries by type of facility and on the number of admissions for sick newborns. Details of the trial protocol, including the surveillance system, have already been published. ${ }^{15}$ The evaluation cohort comprised births occurring between November 2008 and December 2009. ${ }^{15}$

Demand is looked at through an equity lens by stratifying it by socioeconomic quintiles. This is based on an asset index calculated using principal components analysis of a list of household assets collected from women during pregnancy. The asset scores were ranked and divided into quintiles.

\section{Data analysis}

All of the analyses presented in this paper were performed using Stata V.11. Simple and cross tabulations, using the statistical test for associations, were made to obtain 
percentages for the main indicators of the facility survey and to link these with the survey data. For the vignettes, scores obtained from the expert weighting were applied to the responses from the facilities to represent the overall score for each facility. Scatter plots were used to present the overall results and results by each question.

\section{Ethical approval and informed consent}

The Newhints trial was approved by ethical committees at the Kintampo Health Research Centre (KHRC) and the London School of Hygiene and Tropical Medicine (LSHTM). HFA was submitted and approved by the KHRC and LSHTM ethics committees. Informed consent was obtained from all HFA respondents at the time of the interview. Before the start of the Newhints trial, all women of reproductive age living in the trial area consented to the use of their surveillance data. Consent was also derived from women who moved into the trial area at any point during the trial. Information sheets and consent forms were written in the local language and administered by the interviewer to health facility staff and by fieldworkers to women. Respondents were given the right to refuse without detrimental consequences, but there were no refusals. The official record of informed consent was based on a signature for health facility staff and a signature or thumbprint for women.

\section{RESULTS}

\section{Infrastructure indicators}

Table 3 shows the availability of clean water, electricity, a fridge for storage of vaccines, drugs and blood and a sink with soap for hand washing. These were available all the time at the regional, main district and private hospitals, but two of the other district hospitals and the majority of health centres and clinics did not have reliable electricity. Additionally, health centres and clinics did not all have clean water supply or fridges for storage of vaccines. A sink with soap for hand washing was generally available in a majority of the facilities.

\section{Essential equipment for newborn care}

Table 4 presents the availability of essential equipment necessary for postdelivery newborn care. The majority of hospitals had properly functioning resuscitation equipment. The exceptions were one of the main district hospitals and private hospitals that lacked a bag and mask. Overall, maternity homes had better availability of resuscitation-specific equipment than did health centres and clinics. Most facilities, apart from one clinic and one maternity home, had a baby scale to identify very LBW babies. However, one of the four main district hospitals did not have a properly functioning incubator and two did not have cups to measure expressed breast milk. The other four district hospitals and one of the private hospitals lacked these pieces of equipment. Overall, intravenous fluids and infusion sets as well as baby scales were widely available in all facilities.

\section{Essential drugs for sick newborns}

Table 5 shows the availability of intravenous/intramuscular ampicillin and intramuscular gentamicin, first-line antibiotics for newborn sepsis; intravenous diazepam, an anticonvulsant used for mothers and babies; and intramuscular dexamethasone, a drug used primarily in hospitals to prevent breathing problems in premature babies. As can be seen, the regional and main district hospitals had all drugs apart from one main district hospital, which lacked dexamethasone. Other district hospitals only had a complete stock of diazepam while private hospitals lacked only dexamethasone. Diazepam was the only drug that the majority of health centres, clinics and maternity homes stocked while more than $50 \%$ of maternity homes and clinics had gentamicin; less than $40 \%$ of lower level facilities had ampicillin and none had dexamethasone. This is a major shortcoming in any facility performing deliveries.

\section{Profile of human resources for managing sick newborns}

A total of 30 doctors and 44 medical assistants/nurses/ midwives were identified as being capable of managing newborn illness in the four main district hospitals and the other seven facilities where the more detailed HFA was performed. Of these personnel, only one doctor (paediatrician) was professionally trained to deliver newborn care. However, when HFA was conducted, only $23(31 \%)$ of these individuals capable of managing

Table 3 Availability of basic infrastructure in facilities that deliver babies

\begin{tabular}{lccccc}
\hline & \multicolumn{3}{c}{ Always available } \\
\cline { 3 - 5 } Type of facility & Number & Clean water source & Reliable electricity & Fridge for storage & Sink with soap \\
\hline Regional hospital & 1 & $1(100 \%)$ & $1(100 \%)$ & $1(100 \%)$ & $1(100 \%)$ \\
Main district hospital & 4 & $4(100 \%)$ & $4(100 \%)$ & $4(100 \%)$ & $4(100 \%)$ \\
Other district hospital & 4 & $4(100 \%)$ & $2(50 \%)$ & $4(100 \%)$ & $4(100 \%)$ \\
Private hospital & 2 & $2(100 \%)$ & $2(100 \%)$ & $2(100 \%)$ & $2(100 \%)$ \\
Health centre & 34 & $25(74 \%)$ & $2(6 \%)$ & $29(85 \%)$ & $32(94 \%)$ \\
Clinic/CHPS/health post & 8 & $5(63 \%)$ & $1(13 \%)$ & $6(75 \%)$ & $8(100 \%)$ \\
Maternity home & 11 & $11(100 \%)$ & $7(64 \%)$ & $9(82 \%)$ & $9(82 \%)$ \\
Total & 64 & $52(81 \%)$ & $19(30 \%)$ & $55(86 \%)$ & $60(94 \%)$ \\
\hline
\end{tabular}




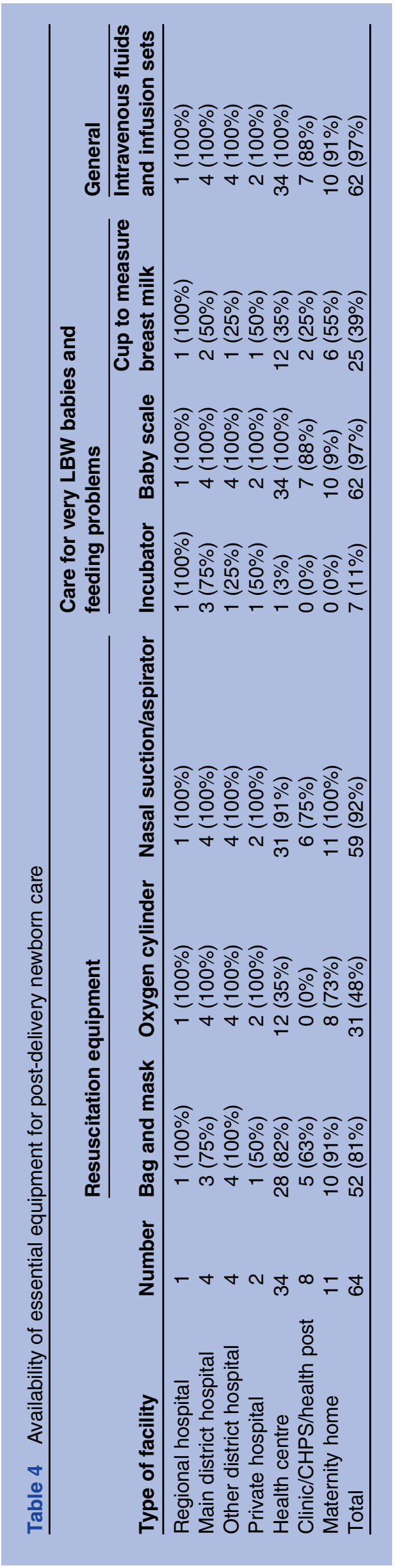

newborn illnesses were present at their posts: 8 (26.7\%) were doctors and $15(34.1 \%)$ medical assistants/nurses/ midwives. None of the doctors in the 11 focus facilities had attended the ENC training conducted before the implementation of the Newhints intervention, whereas $55 \%$ of medical assistants/nurses/midwives capable of managing newborns had attended. However, only $21 \%$ of the latter were at their posts during the assessment. Interviews revealed that some of these individuals were posted to work in different departments of the hospitals where their newborn skills were not being utilised.

\section{Vignette 1: quality of newborn care}

The scores corresponding to each of the three parts related to the ENC vignette plus total score are shown by facility type in figure 2. Only 3 of the 64 facilities, two main district hospitals and one private hospital, scored $80 \%$ or higher overall and were classified as providing a high quality of ENC; $76.6 \%$ (49) achieved low-quality scores. A larger number of facilities (11: 5 hospitals, 3 health centres, 1 clinic and 1 maternity home) scored more than $80 \%$ on part A, life-saving resuscitation. The regional hospital scored less than $80 \%$ for all three parts of the vignette. Only one main district hospital scored over $80 \%$ for parts $\mathrm{B}$ and $\mathrm{C}$ on immediate newborn care and thermal care, respectively, two on immediate resuscitation and two for all three parts combined. Lower level facilities achieved only low-to-moderate scores for the three parts, apart from two maternity homes on part A and one on parts $\mathrm{B}$ and $\mathrm{C}$, and provided overall low quality of ENC.

\section{Vignette 2: quality of care for very LBW babies}

Quality of care for very LBW babies, for the subset of 11 facilities, was slightly better overall than that seen for ENC (figure 3). With respect to the management of very LBW babies, the six hospitals were split between moderate and high-quality scores while most of the lower level facilities, apart from one maternity home, scored low. Quality of care related to feeding was high for three hospitals and two lower-level facilities, and moderate for one hospital and three lower-level facilities.

\section{Delayed discharge for newly delivered babies}

Maternity/newborn ward matrons in the 11 focus facilities generally did poorly in listing the reasons for delaying the discharge of newly delivered babies (table 6). Only 4 of the 13 were mentioned by more than half of the respondents. Three said that they never delayed the discharge of any baby under any circumstances; two of these respondents were from health centres and one from a clinic. In contrast, one respondent was able to list 12 danger signs, missing only 'eye infection'. She was the matron in one of the main referral level hospitals.

Of the 15884 live-births captured through the Newhints surveillance system between November 2008 and December 2009, data were available for 10343 
Table 5 Availability of essential drugs for newborn survival

\begin{tabular}{|c|c|c|c|c|c|}
\hline \multirow[b]{2}{*}{ Type of facility } & \multirow[b]{2}{*}{ Number } & \multicolumn{2}{|c|}{ Management of sepsis } & \multirow{2}{*}{$\begin{array}{l}\begin{array}{l}\text { Managing } \\
\text { convulsions }\end{array} \\
\begin{array}{l}\text { Intravenous } \\
\text { diazepam }\end{array}\end{array}$} & \multirow{2}{*}{$\begin{array}{l}\text { Preventing breathing } \\
\text { problems/neonatal } \\
\text { complications in preterm } \\
\text { deliveries } \\
\begin{array}{l}\text { Intramuscular } \\
\text { dexamethasone }\end{array}\end{array}$} \\
\hline & & $\begin{array}{l}\text { Intravenous/ } \\
\text { intramuscular } \\
\text { ampicillin }\end{array}$ & $\begin{array}{l}\text { Intramuscular } \\
\text { gentamicin }\end{array}$ & & \\
\hline Regional hospital & 1 & $1(100 \%)$ & $1(100 \%)$ & $1(100 \%)$ & $1(100 \%)$ \\
\hline Main district hospital & 4 & $4(100 \%)$ & $4(100 \%)$ & $4(100 \%)$ & $3(75 \%)$ \\
\hline Other district hospital & 4 & $2(50 \%)$ & $3(75 \%)$ & $4(100 \%)$ & $1(25 \%)$ \\
\hline Private hospital & 2 & $2(100 \%)$ & $2(100 \%)$ & $2(100 \%)$ & $0(0 \%)$ \\
\hline Health centre & 34 & $8(24 \%)$ & $14(41 \%)$ & $32(94 \%)$ & $0(0 \%)$ \\
\hline Clinic/CHPS/health post & 8 & $3(38 \%)$ & $6(75 \%)$ & 7 (88\%) & $0(0 \%)$ \\
\hline Maternity home & 11 & $4(36 \%)$ & $3(52 \%)$ & $9(82 \%)$ & $0(0 \%)$ \\
\hline Total & 64 & $24(38 \%)$ & $33(52 \%)$ & $59(92 \%)$ & $5(8 \%)$ \\
\hline
\end{tabular}

babies born in facilities who had survived the first day and who had data on initiation of breastfeeding and delayed bathing. Table 7 shows that large coverage gaps exist for both these immediate newborn care behaviours that should be promoted in all facilities. Overall, only $48.3 \%$ of babies born in facilities were breastfed within $1 \mathrm{~h}$ of birth and bathing was delayed for 6 or more hours in only $42.5 \%$ of them. Delayed bathing for at least $6 \mathrm{~h}$ was highest for babies born in the main district hospitals $(47.8 \%)$, although this ranged from $5.9 \%$ to $68.1 \%$. However, initiation of breastfeeding among those born in the main district hospitals (46\%; range 39.3-58.7\%) lagged behind health centres, private hospitals and other district hospitals. Large gaps in adoption remain.

\section{Assessing demand against quality}

The right-hand side of figure 4 shows where the 15884 live-births occurred between November 2008 and December 2009: $32.1 \%$ were born at home and $67.9 \%$ in health facilities. The majority of facility deliveries occurred at the four main district hospitals $(n=5998$, $37.7 \%$ of all births and $56 \%$ of facility births), followed

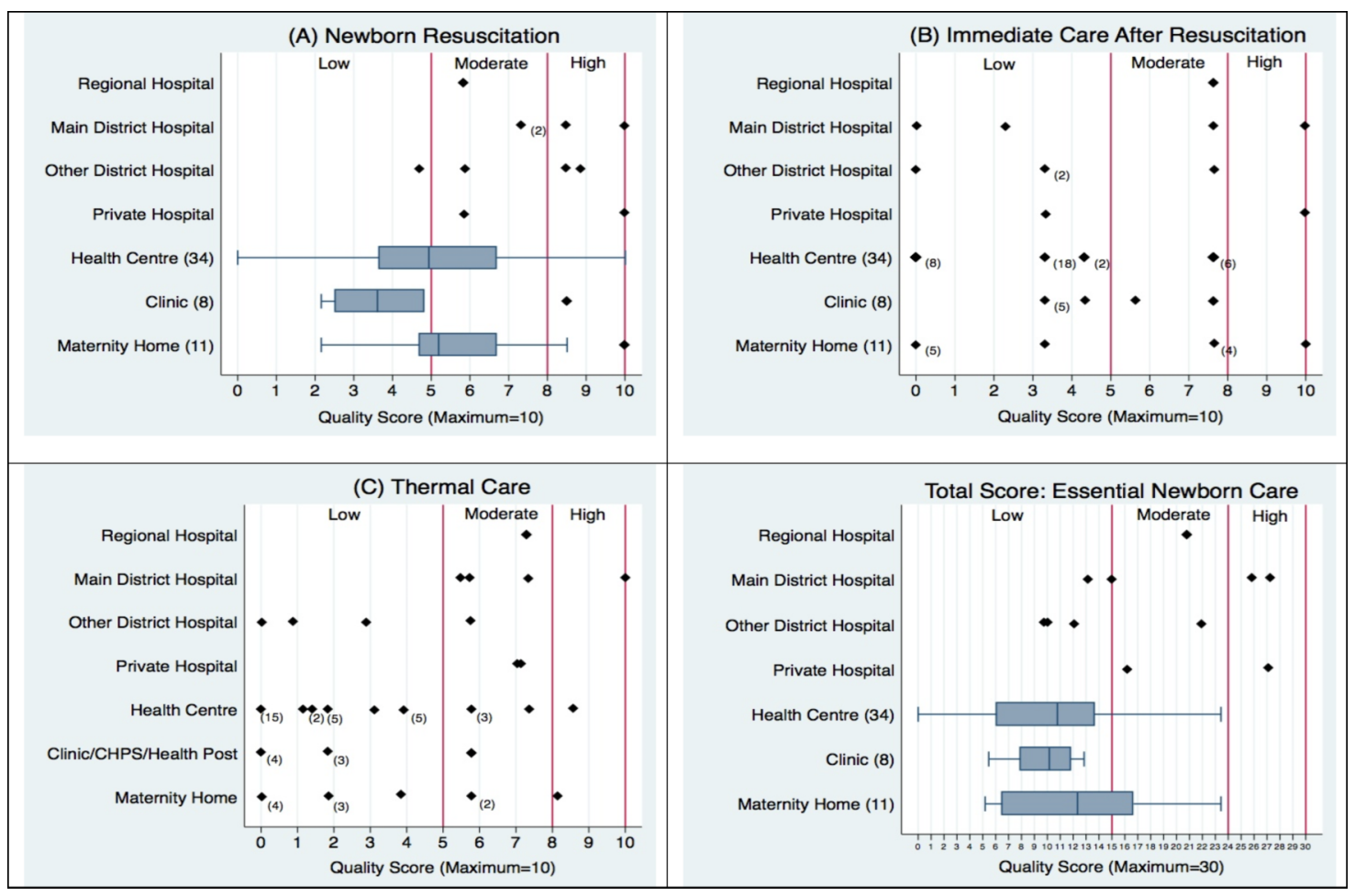

Figure 2 Vignette 1 (essential new-born care) scores by type of facility. The lines in the box plots show the range of scores, while the box captures the range of the middle $50 \%$, with the central line being the median. 


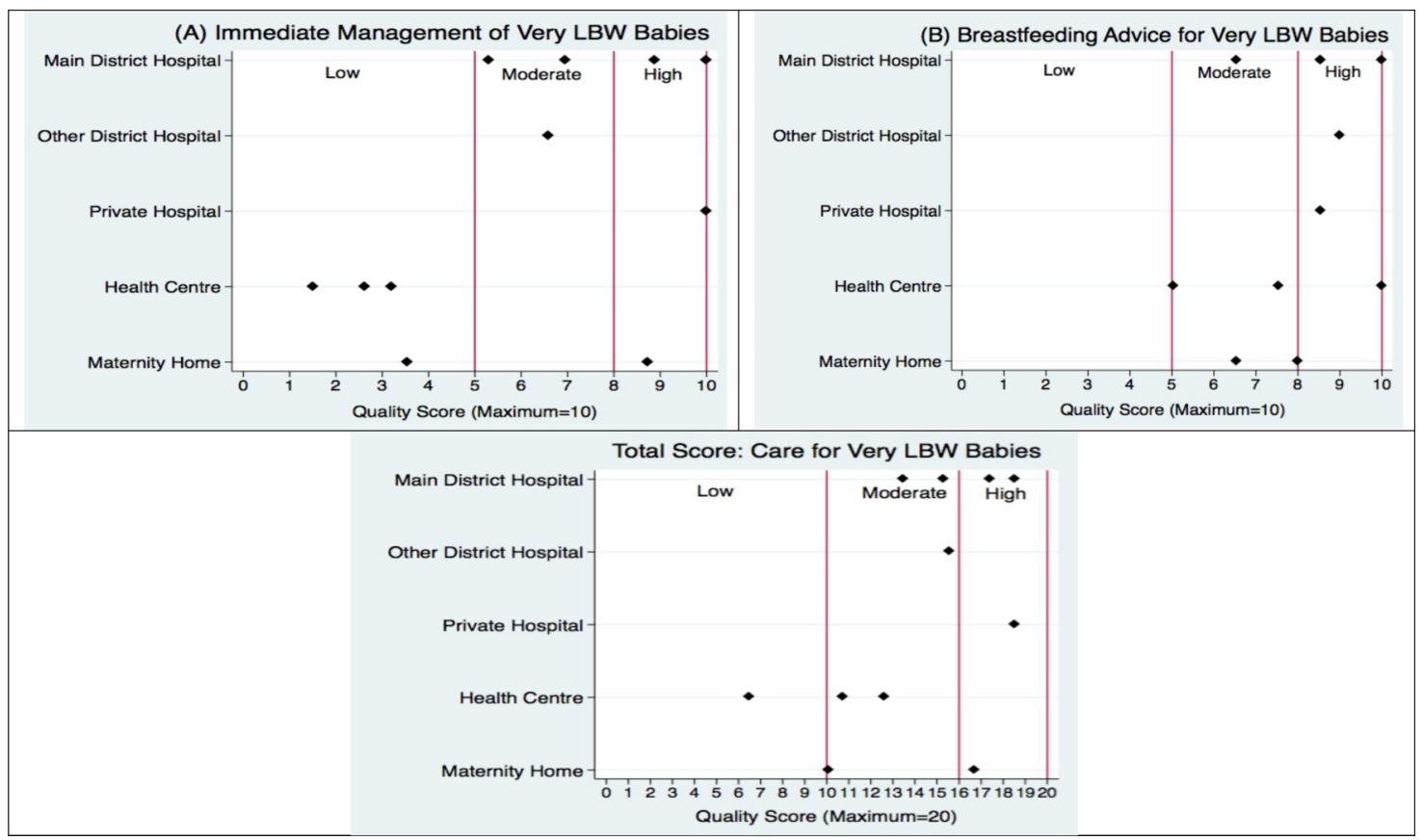

Figure 3 Vignette 2 (care for very low-birth-weight babies) scores by type of facility. The lines in the box plots show the range of scores, while the box captures the range of the middle $50 \%$, with the central line being the median.

by health centres $(\mathrm{n}=2337,14.7 \%)$, maternity homes $(n=1298,8.2 \%)$, other district hospitals $(n=525,3.3 \%)$, clinics $(n=326,2.1 \%)$, private hospitals $(n=226,1.4 \%)$ and the regional hospital $(n=72,0.5 \%)$. Figure 4 also shows that women in lower quintiles were more likely to have home births and less likely to deliver in facilities. It was the wealthier women delivering in the main district hospitals who were provided the best available quality of care for their newborns. There were 98 admissions for ill babies, $85(87 \%)$ of which were made at the main district hospitals with only $4(4.1 \%)$ at the regional hospital.
The majority of facility deliveries and admissions for illness occurred in the four main district hospitals. These facilities possessed the infrastructure necessary to function, and were superior to other facilities, scoring highest for quality of care. However, each of these four hospitals lacked personnel trained in ENC and at least one piece of key equipment or dexamethasone, an essential drug administered to women experiencing preterm labour in order to mature fetal lungs and prevent birth asphyxia in their babies. One hospital capturing 981 births, $9.9 \%$ of which were LBW, lacked both a functioning incubator

Table 6 Reasons for delayed discharge of newly delivered babies by maternity/newborn ward matrons in 11 facilities

\begin{tabular}{|c|c|c|c|c|c|}
\hline \multirow[b]{2}{*}{ Classification } & \multirow[b]{2}{*}{$\begin{array}{l}\text { Reasons for delayed } \\
\text { discharge after birth }\end{array}$} & \multicolumn{4}{|c|}{ Type of health facility } \\
\hline & & Hospital $(n=6)$ & $\begin{array}{l}\text { Health centre* } \\
(n=3)\end{array}$ & $\begin{array}{l}\text { Maternity home* } \\
(\mathrm{n}=2)\end{array}$ & $\begin{array}{l}\text { Total } \\
(n=11)\end{array}$ \\
\hline \multirow{6}{*}{ infection } & Lethargy & $4(66.7 \%)$ & $1(33.3 \%)$ & $1(50.0 \%)$ & $6(54.5 \%)$ \\
\hline & Grunting & $1(16.7 \%)$ & 1 (33.3\%) & $0(0.0 \%)$ & $2(18.2 \%)$ \\
\hline & Breathing difficulty & $1(16.7 \%)$ & 1 (33.3\%) & $0(0.0 \%)$ & $2(18.2 \%)$ \\
\hline & Chest indrawing & 2 (33.3\%) & $0(0.0 \%)$ & $0(0.0 \%)$ & 2 (18.2\%) \\
\hline & Hypothermia & $1(16.7 \%)$ & $0(0.0 \%)$ & $0(0.0 \%)$ & $1(9.1 \%)$ \\
\hline & Fever & $5(83.3 \%)$ & $1(33.3 \%)$ & $0(0.0 \%)$ & $6(54.5 \%)$ \\
\hline \multirow[t]{7}{*}{ Other signs } & Inability to breastfeed & $6(100.0 \%)$ & 1 (33.3\%) & $1(50.0 \%)$ & $8(72.3 \%)$ \\
\hline & Convulsed & $2(33.3 \%)$ & $0(0.0 \%)$ & $0(0.0 \%)$ & $2(18.2 \%)$ \\
\hline & Jaundice & 2 (33.2\%) & $0(0.0 \%)$ & $1(50.0 \%)$ & $3(27.3 \%)$ \\
\hline & Skin pustules & $1(16.7 \%)$ & $0(0.0 \%)$ & $0(0.0 \%)$ & $1(9.1 \%)$ \\
\hline & Eye infection & $0(0.0 \%)$ & $0(0.0 \%)$ & $0(0.0 \%)$ & $0(0.0 \%)$ \\
\hline & Sick & $3(50.0 \%)$ & $1(33.3 \%)$ & $0(0.0 \%)$ & $4(36.4 \%)$ \\
\hline & Very low birth weight & $5(83.3 \%)$ & $1(33.3 \%)$ & $0(0.0 \%)$ & $6(54.5 \%)$ \\
\hline
\end{tabular}


Table 7 Key behaviours by type of facility

\begin{tabular}{lcrr}
\hline Place of delivery & \multicolumn{1}{c}{ Births } & Initiate breastfeeding < $\mathbf{~}$ & Delay bathing $>\mathbf{6} \mathbf{~}$ \\
\hline Regional hospital & $65(0.6 \%)$ & $26(40.0 \%)$ & $21(32.3 \%)$ \\
Main district hospital & $5680(54.9 \%)$ & $2615(46.0 \%)$ & $2715(47.8 \%)$ \\
Other district hospital & $505(4.9 \%)$ & $282(55.8 \%)$ & $171(33.9 \%)$ \\
Private hospital & $216(2.1 \%)$ & $113(52.3 \%)$ & $42(19.4 \%)$ \\
Health centre & $2288(22.1 \%)$ & $1341(58.6 \%)$ & $998(43.6 \%)$ \\
Clinic/CHPS/health post & $320(3.1 \%)$ & $116(36.3 \%)$ & $41(12.8 \%)$ \\
Maternity home & $1269(12.3 \%)$ & $502(39.6 \%)$ & $411(32.4 \%)$ \\
Total & $10343^{*}(100.0 \%)$ & $4995(48.3 \%)$ & $4399(42.5 \%)$ \\
\hline${ }^{*}$ Total number of babies born in facilities who survived the first day and had information on both behaviours.
\end{tabular}

and a bag and mask for resuscitation. Two of the other main district hospitals in which 2234 babies were born (7.1\% LBW) did not have a cup to measure expressed breast milk. And one hospital capturing 2783 births (10\% LBW) did not have a supply of dexamethasone. Overall, none of these hospitals were identified as providing a high quality of immediate and ENC.

Eleven facilities scored highly on quality of immediate newborn resuscitation but two of these, a private hospital and a clinic, did not have a functioning bag and mask. Thus, we estimate that only the 5278 babies born in these nine facilities had access to high-quality, basic resuscitation; this represents $33.2 \%$ of all births. Only one of these (a district hospital) also scored highly on immediate newborn care, as did the private hospital and maternity home; together, they delivered $9.7 \%$ of all babies.Three of the 11 facilities, representing $20.3 \%$ of births, had a high-quality score for the provision of thermal care. Nearly $50 \%$ of facility-born LBW babies were born in the two main district hospitals that received high scores for the quality of care for very LBW babies. Three of these four facilities scored highly on care related to breastfeeding of very LBW babies with all four delaying discharge of newly delivered babies in the presence of feeding problems and a very LBW.

\section{DISCUSSION}

\section{Principal findings}

Nearly $70 \%$ of women delivered in health facilities. Delivery of high-quality newborn care is particularly critical in the main district hospitals since they captured $56 \%$ of facility births and $87 \%$ of neonatal admissions. They possessed the infrastructure necessary to function, which was superior to other facilities. However, almost all facilities lacked certain equipment and drugs; one or more main district hospitals experienced gaps in the availability of incubators, cups to measure breast milk, bag and masks and dexamethasone. Interviews suggested that the main district hospitals did not have adequate staff to manage newborn babies. Additionally, facility respondents in the 11 focus facilities, including hospitals, performed poorly in identifying danger signs that require keeping newborns in hospitals for a longer period. Quality scores for the care of very LBW babies were moderate to high in most facilities. However, only three hospitals achieved a high score overall for quality of ENC; and there were large gaps in the coverage of early initiation of breastfeeding and of delayed bathing for all facility births. This represents a missed opportunity.

\section{Strengths and limitations}

This paper addresses a major evidence gap regarding facility care of newborns in LMICs. The National Health Insurance Scheme's (NHIS) free delivery and newborn care has been operational in the Brong Ahafo region since $2008,{ }^{22-24}$ which has the highest coverage of all regions in Ghana. ${ }^{25}$ NHIS has led to an increase in facility deliveries in the Brong Ahafo region ${ }^{24}$ while the Newhints intervention has substantially increased care-seeking. ${ }^{26}$ This analysis
Figure 4 Live-births by socioeconomic quintile and place of birth in the Newhints cohort.

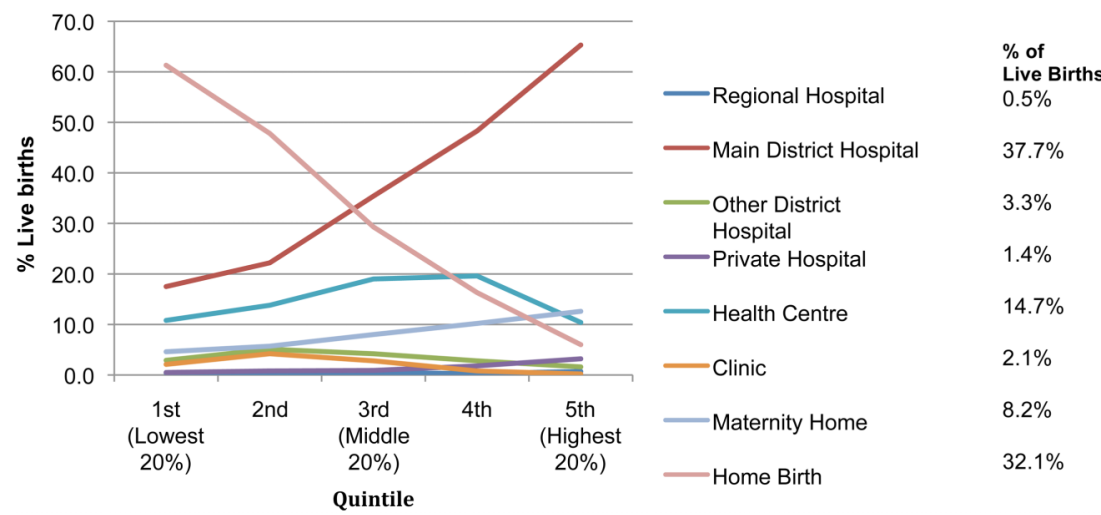


has identified the supply-side components of facility newborn care that need to be strengthened in order to match the demand for services and to increase newborn survival. ${ }^{27}$

A separate paper evaluating the assessment and referral of sick newborns by community volunteers (CBSVs) in the Newhints intervention will describe the health facility response based on in-depth interviews with mothers of referred newborns, CBSVs who referred them and health facility staff. All three groups identified concerns about inadequacies in the quality of care provided to newborns.

HFA was largely based on self-reports. Vignettes were not intended to be clinically complex and comprehensive practicals, but rather as purposely simplified evaluations of crucial, basic newborn care in the first day of life aiming to emphasise the most obvious gaps. They tested the best practice by asking about intended care, which may differ slightly from actual care and could overestimate quality. As vignette interviews were conducted with the highest level nurse/midwife present, results could be interpreted as reflecting the highest quality of care available. Outcome indicators of quality defined by Donabedian ${ }^{20}$ as 'the effects of care on health status of patients', such as neonatal mortality and maternal perceptions of care, were not investigated in this analysis. However, outcome indicators of quality of care are often difficult to evaluate since they can be affected by multiple other factors, including more severe cases being seen at higher level facilities, besides care administered at a health facility.

\section{Comparison to formative research and other studies}

A small HFA, ${ }^{18}$ investigating the capacity of seven facilities in the Brong Ahafo region, was conducted in 2006 as part of the formative research for Newhints ${ }^{15}$; the HFA presented in this paper is considerably more extensive with respect to its content, administration and link with demand. The formative assessment identified gaps in the availability of equipment, inadequate promotion of immediate initiation of breastfeeding and delayed bathing, and the quality of immediate resuscitation. ${ }^{18}$ This HFA shows that little improvement in the capacity and quality of newborn care has been achieved since the formative research. Although facility ENC training was arranged before the implementation of Newhints for staff from the largest 15 facilities, none of the doctors in the main district hospitals attended these training sessions and only a fifth of the medical assistants/nurses/midwives who attended were present at their posts at the time of the HFA; some were no longer caring for newborns. These findings emphasise the critical need for continuous ENC training and retention of trained staff. This needs to be coupled with availability of essential equipment, particularly for LBW babies as facilities tended to have higher scores of quality associated with the care for very LBW babies and delayed discharge, but lacked all the equipment necessary to manage these babies.
Waiswa et $a l^{27}$ also identified poor knowledge of newborn care and availability of proper equipment in Ugandan facilities. Nearly $25 \%$ of first week deaths and $9 \%$ of overall neonatal mortality can be saved with immediate, basic resuscitation using a bag and mask; few babies require advanced resuscitation ${ }^{28-31}$ Bag and masks are inexpensive, simple to use and easy to acquire. ${ }^{29}$ However, Lee $e t a l^{22}$ reported poor quality of neonatal resuscitation in various countries around the world due to lack of proper equipment and trained staff. Although bags and masks were widely available in health facilities in Ghana, low-to-moderate quality scores for immediate newborn resuscitation likely resulted from lack of properly trained staff. We estimated overall that a maximum of $33 \%$ of babies were born in facilities potentially capable of providing high-quality newborn resuscitation; they achieved a high vignette score and had a bag and mask. This is higher than the estimates from Wall et al. ${ }^{33}$ They observed from six African national service provision assessments that only $2-12 \%$ of health workers performing deliveries were trained in newborn resuscitation and 8-22\% had proper equipment available, and concluded that resuscitation was available for less than $25 \%$ of babies and if only about $50 \%$ of women deliver in facilities in many African countries, then accessibility to this life-saving intervention is reduced to about $12.5 \%$ of babies. ${ }^{33}$ Facility training in basic resuscitation in LMICs, the first vital life-saving intervention, can avert about $30 \%$ of intrapartum-related neonatal deaths. ${ }^{33}$

\section{CONCLUSION}

This paper has highlighted major gaps in the availability of ENC equipment and drugs, trained personnel, quality of ENC and provision of care for very LBW babies and promotion in facilities of key ENC practices. Strategies to increase access to facility delivery and care for sick and very LBW babies cannot achieve their potential in saving newborn lives unless they focus on improving the quality of newborn care available at health facilities.

\section{Author affiliations}

${ }^{1}$ Department of Nutrition and Public Health Intervention Research, Faculty of Epidemiology and Population Health, London School of Hygiene and Tropical Medicine, London, UK

${ }^{2}$ Kintampo Health Research Centre, Ghana Health Service, Kintampo, Ghana ${ }^{3}$ Department of Anaesthesiology and Intensive Care Medicine, Jorvi Hospital, Helsinki University Hospital, Espoo, Finland

${ }^{4}$ Institute of Public Health, University of Heidelberg, Heidelberg, Germany ${ }^{5}$ Department of Public Health, Academic Medical Centre, Amsterdam, The Netherlands

${ }^{6}$ Institute of Child Health, University College London, London, UK

Acknowledgements We would like to thank all the health workers who participated in HFA, the mothers who were part of the Newhints trial and the paediatricians and newborn health experts who provided advice and weights for the vignettes.

Contributors LV drafted the paper and conducted the analysis with input, review and approval from all authors. The study was conceived by AM, BRK, AHAtA, ZH, CTA and SO-A who were also responsible for the design and 
conduct of the Newhints trial. HFA was designed by AM, LV, SG and BRK and conducted by TJL, EO, SG and AM. The corresponding author had full access to all the data and, together with the last author, responsibility for the decision to submit for publication. All authors have read and approved the final manuscript.

Funding HFA was funded by WHO, Save the Children's Saving Newborn Lives (SNL) programme from the Bill and Melinda Gates Foundation, and the UK Department of International Development (DFID) for the benefit of developing countries.

Competing interests None.

Ethics approval Ethical committees at the Kintampo Health Research Centre and the London School of Hygiene and Tropical Medicine.

Provenance and peer review Not commissioned; externally peer reviewed.

Data sharing statement No additional data are available.

\section{REFERENCES}

1. WHO. Countdown to 2015 decade report (2000-2010): taking stock of maternal, newborn and child survival. Geneva, Switzerland: World Health Organization, 2010.

2. Lawn JE, Kerber K, Enweronu-Laryea C, et al. 3.6 million neonata deaths - what is progressing and what is not? Semin Perinatol 2010;34:371-86.

3. Oestergaard MZ, Inoue M, Yoshida S, et al. Neonatal mortality levels for 193 countries in 2009 with trends since 1990: a systematic analysis of progress, projections, and priorities. PLoS Med 2011;8: e1001080.

4. Lawn JE, Cousens S, Zupan J. 4 million neonatal deaths: when? Where? Why? Lancet 2005;365:891-900.

5. Darmstadt GL, Bhutta ZA, Cousens S, et al. Evidence-based, cost-effective interventions: how many newborn babies can we save? Lancet 2005;365:977-88

6. UNICEF. Levels \& trends in child mortality: estimates developed by the UN Inter-agency Group for child mortality estimation: United Nations Children's Fund, 2011. http://www.unicef.org/media/files/ Child Mortality_Report_2011_Final.pdf

7. WHO/UNICEF Joint Statement. Home visits for the newborn child a strategy to improve survival, 2009. http://whqlibdoc.who.int/hq/ 2009/WHO_FCH_CAH_09.02_eng.pdf

8. Nolan T, Angos P, Cunha AJ, et al. Quality of hospital care for seriously ill children in less-developed countries. Lancet 2001;357:106-10.

9. Althabe F, Bergel E, Cafferata ML, et al. Strategies for improving the quality of health care in maternal and child health in low- and middle-income countries: an overview of systematic reviews. Paediatr Perinat Epidemiol 2008;22(Suppl 1):42-60.

10. Fauveau V, de Bernis L. 'Good obstetrics' revisited: too many evidence-based practices and devices are not used. Int J Gynaecol Obstet 2006;94:179-84.

11. van den Broek NR, Graham WJ. Quality of care for maternal and newborn health: the neglected agenda. BJOG 2009;116(Suppl 1):18-21.

12. English $\mathrm{M}$, Ntoburi $\mathrm{S}$, Wagai $\mathrm{J}$, et al. An intervention to improve paediatric and newborn care in Kenyan district hospitals: understanding the context. Implementation Sci 2009;4:42.

13. Opondo C, Ntoburi S, Wagai J, et al. Are hospitals prepared to support newborn survival?-an evaluation of eight first-referral level hospitals in Kenya. Trop Med Int Health 2009;14:1165-72.
14. Hozumi D, Fronczak N, Noriega Minichiello S, et al. Profiles of Health Facility Assessment Methods. MEASURE Evaluation. USAID 2006. http://www.cpc.unc.edu/measure/publications/tr-06-36

15. Kirkwood BR, Manu A, Tawiah-Agyemang C, et al. NEWHINTS cluster randomised trial to evaluate the impact on neonatal mortality in rural Ghana of routine home visits to provide a package of essential newborn care interventions in the third trimester of pregnancy and the first week of life: trial protocol. Trials 2010;11:58.

16. Campbell $\mathrm{H}$, Duke $T$, Weber $\mathrm{M}$, et al. Global initiatives for improving hospital care for children: state of the art and future prospects. Pediatrics 2008;121:e984-92.

17. WHO. Integrated management of pregnancy and childbirth Pregnancy, childbirth, postpartum and nerwborn care: a guide for essential practice, 2006. http://whqlibdoc.who.int/publications/2006/ 924159084X_eng.pdf

18. Howe LD, Manu A, Tawiah-Agyemang C, et al. Developing a community-based neonatal care intervention: a health facility assessment to inform intervention design. Paediatr Perinat Epidemiol 2011;25:192-200.

19. Peabody JW, Luck J, Glassman P, et al. Measuring the quality of physician practice by using clinical vignettes: a prospective validation study. Ann Intern Med 2004;141:771-80.

20. Donabedian A. The quality of care. How can it be assessed? JAMA 1988;260:1743-8.

21. Donabedian A. Evaluating the quality of medical care. 1966. Milbank Q 2005;83:691-729.

22. Agyepong IA, Adjei S. Public social policy development and implementation: a case study of the Ghana National Health Insurance scheme. Health Policy Plan 2008;23:150-60.

23. Witter S, Garshong B. Something old or something new? Social health insurance in Ghana. BMC Int Health Hum Rights 2009;9:20.

24. Dzakpasu S, Soremekun S, Manu A, et al. Impact of free delivery care on health facility delivery and insurance coverage in Ghana's Brong Ahafo Region. PLoS One 2012;7:e49430. doi:10.1371/ journal.pone.0049430

25. Ghana Statistical Service (GSS), Ghana Health Service (GHS) Macro I. Ghana Demographic and Health Survey 2008. Accra, Ghana: GSS, GHS and ICF Macro, 2009.

26. Kirkwood BR, Manu A, Ten Asbroek AH, et al. Impact of the 'Newhints' home visits intervention on neonatal mortality and care practices in Ghana: a cluster randomised controlled trial. Lancet 2013 (Apr 8). doi: 10.1016/A0140-6736(13)60055-1

27. Waiswa P, Kallander K, Peterson S, et al. Using the three delays model to understand why newborn babies die in eastern Uganda. Trop Med Int Health 2010;15:964-72.

28. Lawn JE, Wilczynska-Ketende K, Cousens SN. Estimating the causes of 4 million neonatal deaths in the year 2000. Int $J$ Epidemiol 2006;35:706-18.

29. Wall SN, Lee AC, Carlo W, et al. Reducing intrapartum-related neonatal deaths in low- and middle-income countries-what works? Semin Perinatol 2010;34:395-407.

30. WHO. Safe motherhood. Basic newborn resuscitation: a practical guide. Geneva: World Health Organization, 1997.

31. Lawn JE, Kinney M, Lee AC, et al. Reducing intrapartum-related deaths and disability: can the health system deliver? Int J Gynaecol Obstet 2009;107(Suppl 1):S123-40, S40-2.

32. Lee AC, Cousens S, Wall SN, et al. Neonatal resuscitation and immediate newborn assessment and stimulation for the prevention of neonatal deaths: a systematic review, meta-analysis and Delphi estimation of mortality effect. BMC Public Health 2011;11(Suppl 3):S12.

33. Wall SN, Lee AC, Niermeyer $\mathrm{S}$, et al. Neonatal resuscitation in low-resource settings: what, who, and how to overcome challenges to scale up? Int J Gynaecol Obstet 2009;107(Suppl 1):S47-62 S63-4. 